\title{
Preventive Dental Care and Oral Health of Children and Adolescents With and Without Heart Conditions — United States, 2016-2019
}

\author{
Karrie F. Downing, $\mathrm{MPH}^{1}$; Lorena Espinoza, $\mathrm{DDS}^{2}$; Matthew E. Oster, MD ${ }^{1,3}$; Sherry L. Farr, PhD ${ }^{1}$
}

Approximately 900,000 U.S. children have heart conditions, such as congenital heart disease (1). These children might be at increased risk for life-threatening infective endocarditis from oral bacteria in the bloodstream (2). Therefore, preventive dental care (i.e., check-ups, dental cleaning, radiographs, fluoride treatment, or sealant) to maintain oral health is important. Oral health status and receipt of preventive dental care were compared between children with heart conditions $(2,928)$ and without $(116,826)$ using population-based 2016-2019 National Survey of Children's Health (NSCH) data. Approximately $83 \%$ of children with and $80 \%$ without heart conditions received preventive dental care in the past year $(p=0.06)$. Children with heart conditions were more likely than were those without to have poor oral health $(17.2 \%$ versus $13.7 \% ; \mathrm{p}=0.02)$ and teeth in fair or poor condition $(9.9 \%$ versus $5.3 \% ; \mathrm{p}<0.01)$. Among those with a heart condition, having low household income; an intellectual or developmental disability; and no well-child visit or medical home were associated with poor oral health. Receipt of preventive dental care was higher among children aged $\geq 6$ years and those with insurance. Public health practitioners and health care providers can implement strategies (e.g., parent and patient education and collaboration between pediatricians, dentists, and cardiologists) to improve oral health and care among children with heart conditions, especially those with fewer resources and intellectual or developmental disabilities.

The NSCH is an annual parent-reported survey to evaluate health, well-being, and related factors among U.S. persons aged 0-17 years.* One child from each household was randomly selected to be the subject of the survey. The overall weighted response rates per year for the 2016-2019 surveys were 40.7\%, $37.4 \%, 43.1 \%$, and $42.4 \%$, respectively. Parents were asked

\footnotetext{
$\overline{* \text { https://mchb.hrsa.gov/data/national-surveys }}$
}

whether a health care provider ever said their child had a heart condition. Only parents of children and adolescents aged 1-17 years with any teeth were asked about their child's oral health ${ }^{\dagger}$ and

\footnotetext{
†To assess oral health, parents were asked about the condition of their child's teeth and experience with the following indicators of poor oral health during the past 12 months: frequent or chronic difficulty with toothaches, bleeding gums, or decayed teeth or cavities.
}

\section{INSIDE}

196 Progress Toward Rubella and Congenital Rubella Syndrome Control and Elimination - Worldwide, 2012-2020

202 Identifying Higher-Volume Antibiotic Outpatient Prescribers Using Publicly Available Medicare Part D Data — United States, 2019

206 Genomic Surveillance for SARS-CoV-2 Variants: Predominance of the Delta (B.1.617.2) and Omicron (B.1.1.529) Variants — United States, June 2021January 2022

212 Effectiveness of Face Mask or Respirator Use in Indoor Public Settings for Prevention of SARS-CoV-2 Infection - California, February-December 2021

217 Clinical Characteristics and Outcomes Among Adults Hospitalized with Laboratory-Confirmed SARS-CoV-2 Infection During Periods of B.1.617.2 (Delta) and B.1.1.529 (Omicron) Variant Predominance - One Hospital, California, July 15-September 23, 2021, and December 21, 2021-January 27, 2022

224 QuickStats

Continuing Education examination available at https://www.cdc.gov/mmwr/mmwr_continuingEducation.html

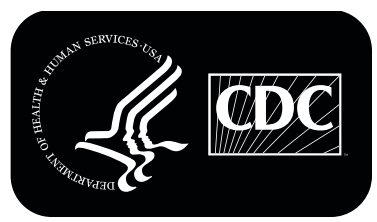


receipt of dental care ${ }^{\S}$. Children missing any data of interest were excluded. Characteristics of excluded and included children and adolescents were compared using Wald chi-square tests. Crude and adjusted associations between heart condition status and oral health and preventive dental care were assessed using Wald chi-square tests and with the predicted marginals obtained from logistic regression models. Among children and adolescents with a heart condition, adjusted prevalence ratios (aPRs) evaluated associations between their characteristics and receipt of preventive dental care, fair or poor condition of teeth, and one or more indicators of poor oral health. All models included sex, age, and race/ethnicity. Certain models also included household income as a percentage of federal poverty level and having intellectual or developmental disabilities, including Down syndrome.

Analyses were conducted using SAS-callable SUDAAN (version 11; RTI International). Design parameters accounting for complex sampling, weight, and nonresponse bias produced nationally representative, population-based estimates. Among 126,996 children and adolescents in NSCH aged $\geq 1$ year who had one or more teeth, $6.3 \%$ were excluded because information on heart condition $(0.3 \%)$, oral or dental outcomes

\footnotetext{
${ }^{\$}$ To assess receipt of preventive dental care, parents were first asked if the child saw a dentist or oral health care provider for any oral health care during the past 12 months. If yes, they were asked if the child saw the oral health care provider for preventive dental care. Among children who received preventive dental care, the following were examined: check-ups, cleaning, instruction on tooth brushing and oral health care, radiographs, fluoride treatment, or sealant.
}

(2.9\%), or other characteristics (3.1\%) was missing; although there was no difference in heart condition status between included and excluded persons, excluded persons more commonly had poor oral health and less commonly had preventive dental visits. In total, 2,928 children and adolescents with heart conditions (representing 1.4 million U.S. children and adolescents) and 116,826 children and adolescents without heart conditions (representing 6.4 million U.S. children and adolescents) were included.

Children and adolescents with heart conditions were less likely to be Hispanic or uninsured and more likely to be non-Hispanic White and have public insurance, intellectual or developmental disabilities, special health care needs, and well-child visits (Table 1 ). Approximately $84 \%$ of those with a heart condition received any dental care in the past 12 months (K Downing, CDC, unpublished data, 2022), and 83\% received preventive dental care (Figure). Among children and adolescents who received preventive dental care, the majority received dental check-ups and dental cleanings (95\% each), whereas application of sealant was least common (25\%). Children and adolescents with a heart condition were more likely than were those without to receive preventive dental care overall as well as each of the individual services, although some CIs overlapped. Children and adolescents with a heart condition were approximately twice as likely to have teeth in fair or poor condition $(10 \%)$ as were those without a heart condition (5\%). Seventeen percent of children and adolescents

The MMWR series of publications is published by the Center for Surveillance, Epidemiology, and Laboratory Services, Centers for Disease Control and Prevention (CDC), U.S. Department of Health and Human Services, Atlanta, GA 30329-4027.

Suggested citation: [Author names; first three, then et al., if more than six.] [Report title]. MMWR Morb Mortal Wkly Rep 2022;71:[inclusive page numbers].

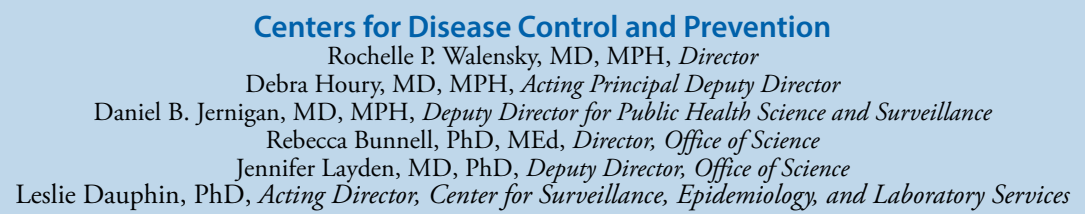

MMWR Editorial and Production Staff (Weekly)

Charlotte K. Kent, PhD, MPH, Editor in Chief Jacqueline Gindler, MD, Editor

Paul Z. Siegel, MD, MPH, Associate Editor

Mary Dott, MD, MPH, Online Editor

Terisa F. Rutledge, Managing Editor

Teresa M. Hood, MS, Lead Technical Writer-Editor

Leigh Berdon, Glenn Damon, Soumya Dunworth, PhD

Tiana Garrett-Cherry, PhD, MPH, Srila Sen, MA, Stacy Simon, MA, Morgan Thompson,

Technical Writer-Editors

Matthew L. Boulton, MD, MPH

Carolyn Brooks, ScD, MA

Jay C. Butler, MD

Virginia A. Caine, MD

Jonathan E. Fielding, MD, MPH, MBA

David W. Fleming, MD
Martha F. Boyd, Lead Visual Information Specialist

Alexander J. Gottardy, Maureen A. Leahy,

Julia C. Martinroe, Stephen R. Spriggs, Tong Yang, Visual Information Specialists

Quang M. Doan, MBA, Phyllis H. King,

Terraye M. Starr, Moua Yang,

Information Technology Specialists
Ian Branam, MA

Acting Lead Health Communication Specialist Shelton Bartley, MPH, Leslie Hamlin,

Lowery Johnson, Amanda Ray,

Health Communication Specialists

Will Yang, MA,

Visual Information Specialist
MMWR Editorial Board

Timothy F. Jones, MD, Chairman

William E. Halperin, MD, DrPH, MPH

Jewel Mullen, MD, MPH, MPA

Jeff Niederdeppe, $\mathrm{PhD}$

Celeste Philip, MD, MPH

Patricia Quinlisk, MD, MPH

Patrick L. Remington, MD, MPH
Carlos Roig, MS, MA

William Schaffner, MD

Nathaniel Smith, MD, MPH

Morgan Bobb Swanson, BS

Abbigail Tumpey, MPH 
TABLE 1. Characteristics of persons aged 1-17 years with and without a heart condition — National Survey of Children's Health,* United States, 2016-2019

\begin{tabular}{|c|c|c|c|c|}
\hline \multirow[b]{3}{*}{ Characteristic } & \multicolumn{4}{|c|}{ Parental report of a heart condition } \\
\hline & \multicolumn{2}{|c|}{$\begin{array}{c}\text { Never } \\
(n=116,826)\end{array}$} & \multicolumn{2}{|c|}{$\begin{array}{c}\text { Ever } \\
(\mathrm{n}=2,928)\end{array}$} \\
\hline & No. & $\begin{array}{l}\text { Weighted \% } \\
\text { (95\% CI) }\end{array}$ & No. & $\begin{array}{l}\text { Weighted \% } \\
\text { (95\% Cl) }\end{array}$ \\
\hline \multicolumn{5}{|l|}{ Sex } \\
\hline Male & 60,398 & $51.1(50.4-51.7)$ & 1,607 & $52.5(48.8-56.3)$ \\
\hline Female & 56,428 & $48.9(48.3-49.6)$ & 1,321 & $47.5(43.7-51.2)$ \\
\hline \multicolumn{5}{|l|}{ Age group, yrs } \\
\hline $1-5$ & 30,304 & $28.6(28.0-29.2)$ & 715 & $26.7(23.6-29.9)$ \\
\hline $6-11$ & 36,866 & $35.6(35.0-36.3)$ & 914 & $37.7(33.9-41.6)$ \\
\hline $12-17$ & 49,656 & $35.8(35.2-36.4)$ & 1,299 & $35.7(32.3-39.1)$ \\
\hline \multicolumn{5}{|l|}{ Race/Ethnicity } \\
\hline Black, non-Hispanic & 7,024 & $13.1(12.6-13.6)$ & 175 & $12.7(10.3-15.6)$ \\
\hline White, non-Hispanic & 81,661 & $51.3(50.6-51.9)$ & 2,141 & $60.1(56.2-63.9)$ \\
\hline Hispanic & 13,284 & $24.9(24.2-25.6)$ & 279 & $17.8(14.5-21.6)$ \\
\hline Multiracial/Other ${ }^{\dagger}$ & 14,857 & $10.7(10.4-11.0)$ & 333 & $9.5(7.6-11.7)$ \\
\hline \multicolumn{5}{|l|}{ Insurance coverage } \\
\hline Any private & 89,487 & $63.3(62.6-64.0)$ & 2,119 & $59.7(55.6-63.6)$ \\
\hline Public only & 22,520 & $30.3(29.6-31.0)$ & 717 & $36.3(32.3-40.4)$ \\
\hline None & 4,819 & $6.4(6.0-6.8)$ & 92 & $4.1(2.8-5.8)$ \\
\hline \multicolumn{5}{|l|}{ Federal poverty level ${ }^{\S}$} \\
\hline$<100 \%$ & 12,483 & $19.6(19.0-20.2)$ & 370 & $23.4(19.6-27.8)$ \\
\hline $100 \%-199 \%$ & 18,527 & $21.7(21.1-22.3)$ & 493 & $19.3(16.5-22.3)$ \\
\hline $200 \%-399 \%$ & 36,185 & $27.6(27.1-28.2)$ & 959 & $29.1(26.1-32.3)$ \\
\hline$\geq 400 \%$ & 49,631 & $31.1(30.6-31.6)$ & 1,106 & $28.3(25.5-31.2)$ \\
\hline Has an intellectual or developmental disability & 7,776 & $6.5(6.2-6.8)$ & 697 & $24.2(21.4-27.3)$ \\
\hline Has special health care needs $\boldsymbol{q}^{\natural}$ & 27,000 & $19.2(18.7-19.7)$ & 1,533 & $47.9(44.2-51.6)$ \\
\hline Attended well-child visit ${ }^{* *}$ & 96,817 & $79.6(79.0-80.1)$ & 2,639 & $89.6(87.1-91.6)$ \\
\hline Has a medical home $^{t \dagger}$ & 64,104 & $48.6(47.9-49.2)$ & 1,555 & $48.7(44.9-52.4)$ \\
\hline
\end{tabular}

\footnotetext{
* The National Survey of Children's Health is weighted to be representative of the U.S. population of noninstitutionalized persons aged $\leq 17$ years. https://www2. census.gov/programs-surveys/nsch/technical-documentation/methodology/NSCH-Guide-to-Multi-Year-Estimates.pdf

$\dagger$ "Other" category includes respondents who self-identified as Asian, American Indian or Alaska Native, Native Hawaiian or other Pacific Islander, or mixed race.

$\S$ Based on U.S. Department of Health and Human Services poverty guidelines.

I Special health care needs are defined as one or more of the following five conditions: needing prescription medicine; having more health care encounters than other children their age; having limitations compared with other children their age; needing physical, occupational, or speech therapy; or having an emotional, developmental, or behavioral problem in need of counseling or treatment. To be classified as special health care needs, these conditions must be related to a medical, behavioral, emotional, developmental, or other health condition that lasts or is expected to last $\geq 12$ months.

** Attended a well-child visit in the past 12 months.

${ }^{\dagger+}$ https://www.childhealthdata.org/docs/medical-home/mhmanual_withappendices-updated-12-7-10-pdf
}

with a heart condition had one or more indicators of poor oral health during the past 12 months. Decayed teeth or cavities (14\%) was the most prevalent indicator of poor oral health. Prevalence for all indicators was higher among children and adolescents with a heart condition than among those without, although some CIs overlapped.

The adjusted prevalence of receipt of preventive dental care was similar in children and adolescents with and without a heart condition (Table 2). Among those with a heart condition, receipt of preventive dental care was more prevalent among older age groups than youngest $(6-11$ years: $\mathrm{aPR}=1.3$; 12-17 years: $\mathrm{aPR}=1.3)$. Point prevalence of preventive dental care was lowest among those without health insurance $(55.7 \%$; aPR $=0.6)$. Prevalence was also lower among those without a medical home $(\mathrm{aPR}=0.9)$.

Children and adolescents with a heart condition were more likely to have teeth in fair or poor condition $(\mathrm{aPR}=1.8)$ and to have one or more poor oral health indicators $(\mathrm{aPR}=1.2)$ than were those without a heart condition (Table 2). For both children and adolescents with teeth in fair or poor condition $(\mathrm{aPR}=1.4)$ and those with one or more poor oral health indicators $(\mathrm{aPR}=1.1)$, results were attenuated, but the former remained elevated, after adjusting for presence of intellectual disabilities (K Downing, CDC, unpublished data, 2022). Among children and adolescents

\footnotetext{
Shttps://www.childhealthdata.org/docs/medical-home/ mhmanual_withappendices-updated-12-7-10-pdf
} 
FIGURE. Weighted prevalence* of preventive dental care and oral health indicators ${ }^{\dagger}$ of persons aged 1-17 years with and without a heart condition — National Survey of Children's Health, United States, 2016-2019

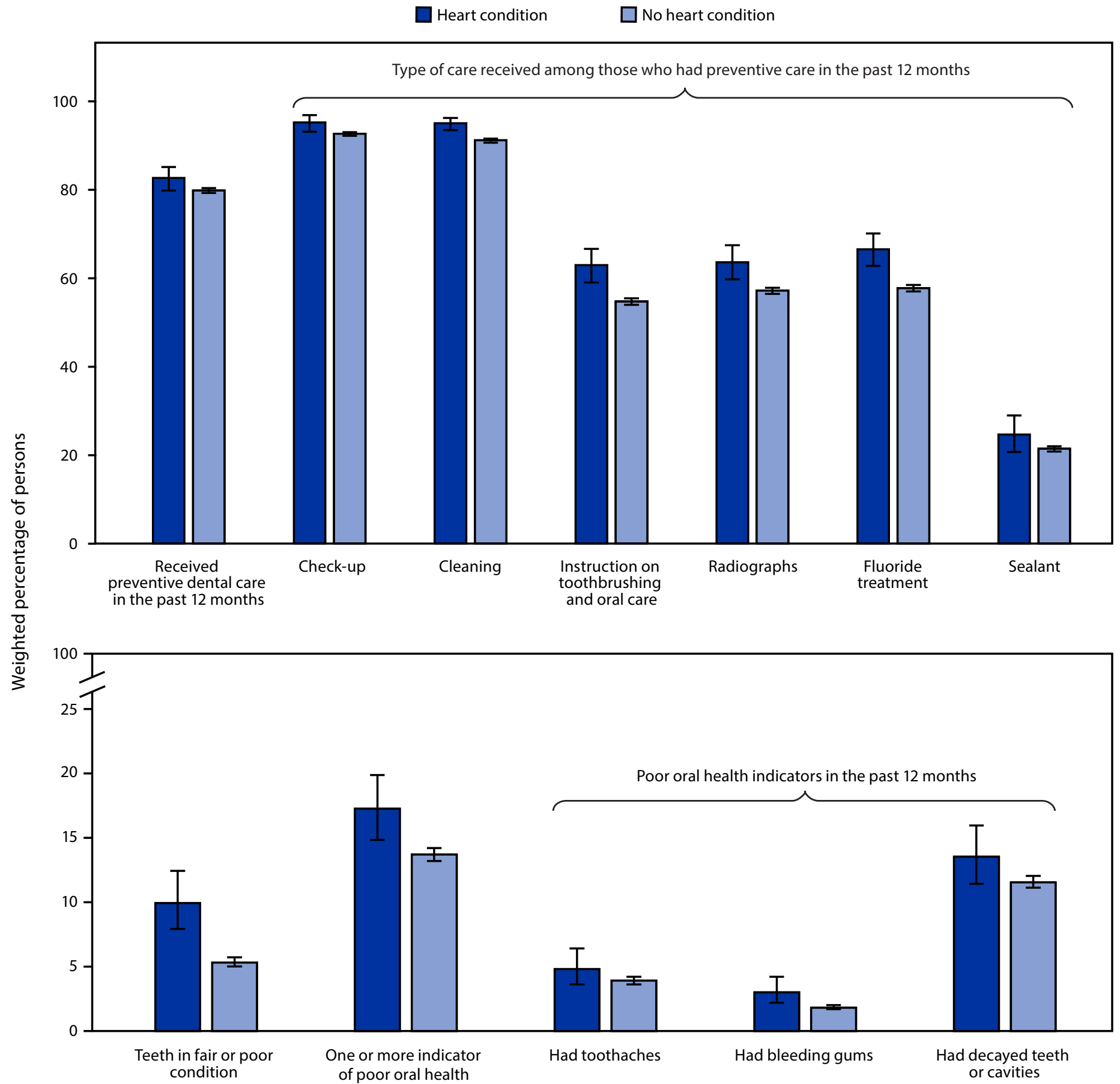

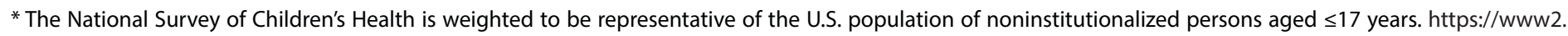
census.gov/programs-surveys/nsch/technical-documentation/methodology/NSCH-Guide-to-Multi-Year-Estimates.pdf

† Parent reported that child had frequent or chronic difficulty with toothaches, bleeding gums, decayed teeth, or cavities in the past 12 months. 
TABLE 2. Associations between oral health and dental care and ever having a heart condition among persons aged 1-17 years with and without heart conditions and associations with sociodemographic and health characteristics among persons with a heart condition - National Survey of Children's Health,* United States, 2016-2019

\begin{tabular}{|c|c|c|c|c|c|c|}
\hline \multirow[b]{2}{*}{ Characteristic } & \multicolumn{2}{|c|}{$\begin{array}{c}\text { Received preventive dental care in } \\
\text { the past } 12 \text { months }\end{array}$} & \multicolumn{2}{|c|}{$\begin{array}{l}\text { Teeth in fair or } \\
\text { poor condition }\end{array}$} & \multicolumn{2}{|c|}{$\begin{array}{c}\text { One or more indicator of } \\
\text { poor oral health }{ }^{\dagger}\end{array}$} \\
\hline & $\begin{array}{l}\text { Weighted \% } \\
\quad(95 \% \mathrm{Cl})\end{array}$ & $\begin{array}{c}\mathrm{aPR}^{\S} \\
(95 \% \mathrm{Cl})\end{array}$ & $\begin{array}{l}\text { Weighted \% } \\
\quad(95 \% \mathrm{Cl})\end{array}$ & $\begin{array}{c}\mathrm{aPR}^{\S} \\
(95 \% \mathrm{Cl})\end{array}$ & $\begin{array}{l}\text { Weighted \% } \\
\quad(95 \% \mathrm{Cl})\end{array}$ & $\begin{array}{c}\mathrm{aPR}^{\S} \\
(95 \% \mathrm{Cl})\end{array}$ \\
\hline \multicolumn{7}{|c|}{ Among all persons $(\mathrm{N}=119,754)$} \\
\hline $\begin{array}{l}\text { Heart condition? } \\
\text { Ever } \\
\text { Never }\end{array}$ & $\begin{array}{l}82.6(79.8-85.1) \\
79.8(79.2-80.3)\end{array}$ & $\begin{array}{l}1.0(1.0-1.1) \\
\quad \text { Ref }\end{array}$ & $\begin{array}{r}9.9(7.9-12.4) \\
5.3(5.0-5.7)\end{array}$ & $\begin{array}{l}1.8(1.4-2.3) \\
\quad \text { Ref }\end{array}$ & $\begin{array}{l}17.2(14.8-19.8) \\
13.7(13.2-14.2)\end{array}$ & $\begin{array}{c}1.2(1.0-1.4) \\
\text { Ref }\end{array}$ \\
\hline \multicolumn{7}{|c|}{ Among persons with a heart condition $(n=2,928)$} \\
\hline $\begin{array}{l}\text { Sex }{ }^{\natural, * *} \\
\text { Male } \\
\text { Female }\end{array}$ & $\begin{array}{l}80.3(76.1-83.9) \\
85.1(81.3-88.2)\end{array}$ & $\begin{array}{c}\text { Ref } \\
1.0(1.0-1.1)\end{array}$ & $\begin{array}{r}11.8(8.8-15.7) \\
7.9(5.6-11.1)\end{array}$ & $\begin{array}{c}\text { Ref } \\
0.9(0.6-1.3)\end{array}$ & $\begin{array}{l}17.0(14.0-20.5) \\
17.3(13.8-21.5)\end{array}$ & $\begin{array}{c}\text { Ref } \\
1.1(0.8-1.5)\end{array}$ \\
\hline $\begin{array}{l}\text { Age group, yrs } \\
1-5 \\
6-11 \\
12-17\end{array}$ & $\begin{array}{l}66.4(60.1-72.2) \\
90.8(86.4-93.9) \\
86.0(81.3-89.6)\end{array}$ & $\begin{array}{c}\text { Ref } \\
1.3(1.2-1.5) \\
1.3(1.1-1.4)\end{array}$ & $\begin{array}{r}9.7(5.9-15.7) \\
9.5(6.5-13.7) \\
10.6(7.6-14.6)\end{array}$ & $\begin{array}{c}\text { Ref } \\
1.2(0.7-2.0) \\
1.3(0.8-2.1)\end{array}$ & $\begin{array}{l}14.7(10.4-20.3) \\
18.2(14.3-22.9) \\
17.9(14.3-22.2)\end{array}$ & $\begin{array}{c}\text { Ref } \\
1.3(0.9-1.9) \\
1.2(0.8-1.9)\end{array}$ \\
\hline $\begin{array}{l}\text { Race/Ethnicity }{ }^{\text {Il,** }} \\
\text { Black, non-Hispanic } \\
\text { White, non-Hispanic } \\
\text { Hispanic } \\
\text { Multiracial/Other }{ }^{\dagger \dagger}\end{array}$ & $\begin{array}{l}77.9(68.6-85.0) \\
83.4(80.2-86.3) \\
88.5(81.6-93.1) \\
72.3(59.3-82.3)\end{array}$ & $\begin{array}{l}1.0(0.9-1.1) \\
\quad \text { Ref } \\
1.0(1.0-1.1) \\
0.9(0.8-1.0)\end{array}$ & $\begin{array}{r}16.5(9.1-28.0) \\
8.5(6.5-11.1) \\
6.8(3.6-12.2) \\
16.3(8.4-29.2)\end{array}$ & $\begin{array}{c}1.1(0.6-1.9) \\
\quad \text { Ref } \\
0.8(0.4-1.5) \\
1.7(0.9-3.1)\end{array}$ & $\begin{array}{l}24.3(16.1-34.9) \\
13.5(11.3-16.2) \\
22.2(15.1-31.2) \\
21.1(14.5-29.8)\end{array}$ & $\begin{array}{c}1.5(0.9-2.3) \\
\quad \text { Ref } \\
1.5(1.0-2.3) \\
1.5(1.0-2.2)\end{array}$ \\
\hline $\begin{array}{l}\text { Insurance coverage* } \\
\text { Any private } \\
\text { Public only } \\
\text { None }\end{array}$ & $\begin{array}{l}85.5(82.6-88.0) \\
80.8(75.1-85.4) \\
55.7(36.8-73.1)\end{array}$ & $\begin{array}{c}\text { Ref } \\
1.0(0.9-1.0) \\
0.6(0.5-0.9)\end{array}$ & $\begin{array}{r}7.8(6.0-10.2) \\
11.7(8.0-16.9) \\
25.1(10.0-50.4)\end{array}$ & $\begin{array}{c}\text { Ref } \\
1.0(0.7-1.5) \\
2.5(1.1-5.4)\end{array}$ & $\begin{array}{c}15.9(13.1-19.2) \\
19.4(15.1-24.5) \\
15.7(7.5-30.0)\end{array}$ & $\begin{array}{c}\text { Ref } \\
1.0(0.7-1.4) \\
0.8(0.4-1.7)\end{array}$ \\
\hline $\begin{array}{l}\text { Federal poverty leve } \\
<100 \% \\
100 \%-199 \% \\
200 \%-399 \% \\
\geq 400 \%\end{array}$ & $\begin{array}{l}78.6(70.3-85.1) \\
81.0(74.7-86.0) \\
83.9(78.9-87.8) \\
85.7(81.1-89.3)\end{array}$ & $\begin{array}{l}0.9(0.8-1.0) \\
0.9(0.9-1.0) \\
1.0(0.9-1.1) \\
\quad \text { Ref }\end{array}$ & $\begin{array}{r}20.0(13.2-29.1) \\
7.4(4.9-11.0) \\
7.9(5.5-11.4) \\
5.5(3.6-8.2)\end{array}$ & $\begin{array}{l}2.9(1.7-4.9) \\
1.2(0.7-2.1) \\
1.4(0.9-2.4) \\
\quad \text { Ref }\end{array}$ & $\begin{array}{c}23.1(16.8-31.0) \\
18.5(13.3-25.2) \\
17.1(13.3-21.7) \\
11.4(8.4-15.3)\end{array}$ & $\begin{array}{c}1.7(1.1-2.6) \\
1.4(0.9-2.2) \\
1.4(1.0-2.1) \\
\quad \text { Ref }\end{array}$ \\
\hline $\begin{array}{l}\text { Has an intellectual o } \\
\text { Yes } \\
\text { No }\end{array}$ & $\begin{array}{l}\text { disability }{ }^{\uparrow, * *} \\
78.3(71.8-83.7) \\
84.0(80.9-86.6)\end{array}$ & $\begin{array}{l}1.0(0.9-1.1) \\
\quad \text { Ref }\end{array}$ & $\begin{array}{r}25.8(19.9-32.8) \\
4.9(3.3-7.0)\end{array}$ & $\begin{array}{l}4.7(3.0-7.4) \\
\quad \text { Ref }\end{array}$ & $\begin{array}{l}25.2(20.0-31.2) \\
14.6(12.1-17.5)\end{array}$ & $\begin{array}{c}1.7(1.3-2.3) \\
\quad \operatorname{Ref}\end{array}$ \\
\hline $\begin{array}{l}\text { Has special health ca } \\
\text { Yes } \\
\text { No }\end{array}$ & $\begin{array}{l}81.8(77.4-85.6) \\
83.3(79.6-86.4)\end{array}$ & $\begin{array}{l}1.0(0.9-1.1) \\
\quad \text { Ref }\end{array}$ & $\begin{array}{r}15.1(11.5-19.5) \\
5.2(3.5-7.7)\end{array}$ & $\begin{array}{l}1.2(0.7-2.1) \\
\quad \text { Ref }\end{array}$ & $\begin{array}{l}20.7(17.3-24.7) \\
13.9(10.9-17.6)\end{array}$ & $\begin{array}{l}1.2(0.8-1.6) \\
\text { Ref }\end{array}$ \\
\hline $\begin{array}{l}\text { Attended well-child } \\
\text { Yes } \\
\text { No }\end{array}$ & $\begin{array}{l}83.5(80.6-86.0) \\
75.0(63.2-84.0)\end{array}$ & $\begin{array}{c}\text { Ref } \\
0.9(0.8-1.0)\end{array}$ & $\begin{array}{r}8.9(7.1-11.1) \\
19.1(9.7-34.2)\end{array}$ & $\begin{array}{c}\text { Ref } \\
1.9(1.2-3.2)\end{array}$ & $\begin{array}{l}16.9(14.5-19.7) \\
19.4(12.3-29.1)\end{array}$ & $\begin{array}{c}\text { Ref } \\
1.1(0.7-1.7)\end{array}$ \\
\hline $\begin{array}{l}\text { Has a medical home } \\
\text { Yes } \\
\text { No }\end{array}$ & $\begin{array}{l}87.9(85.1-90.3) \\
77.5(72.9-81.6)\end{array}$ & $\begin{array}{c}\text { Ref } \\
0.9(0.8-0.9)\end{array}$ & $\begin{array}{r}5.6(4.0-7.9) \\
14(10.6-18.4)\end{array}$ & $\begin{array}{c}\text { Ref } \\
1.9(1.3-2.9)\end{array}$ & $\begin{array}{l}16.2(13.1-20.0) \\
18.0(14.7-21.9)\end{array}$ & $\begin{array}{c}\text { Ref } \\
1.0(0.7-1.3)\end{array}$ \\
\hline
\end{tabular}

Abbreviations: $\mathrm{aPR}=$ adjusted prevalence ratio; Ref $=$ referent group.

* The National Survey of Children's Health is weighted to be representative of the U.S. population of noninstitutionalized persons aged $\leq 17$ years. https://www2. census.gov/programs-surveys/nsch/technical-documentation/methodology/NSCH-Guide-to-Multi-Year-Estimates.pdf

† Parent reported that child had frequent or chronic difficulty with toothaches, bleeding gums, decayed teeth, or cavities in the past 12 months.

$\S$ Multivariable model includes sex, age, and race/ethnicity.

"Model also includes percentage of the federal poverty level.

** Model also includes having intellectual or developmental disabilities.

t† "Other" category includes respondents who self-identified as Asian, American Indian or Alaska Native, Native Hawaiian or other Pacific Islander, or mixed race.

$\S \S$ Based on U.S. Department of Health and Human Services poverty guidelines.

१ी Defined as having one or more of the following five conditions: needing prescription medicine, having more health care encounters than other children their age; having limitations compared with other children their age; needing physical, occupational, or speech therapy or having an emotional, developmental, or behavioral problem in need of counseling or treatment. To be classified as special health care needs, these conditions must be related to a medical, behavioral, emotional, developmental, or other health condition that lasts or is expected to last $\geq 12$ months.

*** Attended a well-child visit in the past 12 months.

${ }^{++\dagger}$ https://www.childhealthdata.org/docs/medical-home/mhmanual_withappendices-updated-12-7-10-pdf 
with a heart condition, the prevalence of having teeth in fair or poor condition was highest among those with intellectual or developmental disabilities $(25.8 \%$; aPR $=4.7)$, those without insurance $(25.1 \%$; $\mathrm{aPR}=2.5)$, and those living at $<100 \%$ of the federal poverty level $(20.0 \%$; aPR $=2.9)$. This prevalence was elevated among persons without well-child visits $(\mathrm{aPR}=1.9)$ and without a medical home $(\mathrm{aPR}=1.9)$. The percentage of children and adolescents with one or more poor oral health indicators was highest among those with an intellectual or developmental disability $(25.2 \% ; \mathrm{aPR}=1.7)$ and was elevated among those living at $<100 \%$ of the federal poverty level $(\mathrm{aPR}=1.7)$.

\section{Discussion}

In this large, population-based sample from the 2016-2019 $\mathrm{NSCH}$, approximately $10 \%$ of children and adolescents with a heart condition had teeth in fair or poor condition, and $17 \%$ had one or more indicators of poor oral health, such as toothaches, bleeding gums, or cavities in the past 12 months. Furthermore, one in six had not received preventive dental care in the past 12 months. Prevalence of preventive dental care was consistently higher among children with a heart condition than among children without, although some differences did not reach statistical significance. Prevalence of poor oral health was also higher among children with a heart condition, although some differences were not statistically significant.

Some small, non-U.S., clinic-based studies have reported that children with congenital heart defects have worse oral health than children without heart defects (3-6), whereas others suggest no difference $(7,8)$. Factors associated with preventive dental care and oral health among children with a heart condition have been less studied. In a $2016 \mathrm{NSCH}$ analysis among all U.S. children, preventive dental care was similarly associated with older age and having insurance (9). Better condition of teeth was associated with well-child visits, although not with household income. In other literature, children with intellectual or developmental disabilities (who account for approximately one in five children with heart conditions in $\mathrm{NSCH}$ ) had some of the highest rates of poor oral health (10).

The findings in this report are subject to at least five limitations. First, all data were parent-reported and not clinically confirmed. Second, sample size limited the ability to examine outcomes by heart condition severity, and data on heart condition type (congenital or acquired) were not collected. Third, some children might have had heart conditions in the past that were resolved. Fourth, $6 \%$ of surveys were excluded for missing data but are not expected to affect findings. Finally, only data from 2019 and earlier were available at the time of analysis, and receipt of dental treatment and oral health might have changed since then.

\section{Summary}

What is already known about this topic?

U.S. children with heart conditions might be at increased risk for infective endocarditis from oral bacteria; however, little is known about their oral health.

What is added by this report?

During 2016-2019, only 83\% of persons aged 1-17 years with heart conditions received preventive dental care. However, $17 \%$ had symptoms of poor oral health during a 12-month period, and $10 \%$ had teeth in fair or poor condition. Those with lower household incomes and intellectual and developmental disabilities had worse oral health.

What are the implications for public health practice?

Public health practitioners and health care providers can implement strategies to improve oral health and care among children with heart conditions, especially those with fewer resources and intellectual or developmental disabilities.

Children and adolescents with a heart condition, particularly those with intellectual disabilities, were more likely than those without a heart condition to have teeth in fair or poor condition. Approximately one in six children with a heart condition had toothaches, bleeding gums, or decay, and approximately one in six had not received preventive dental care during the past 12 months; although rates of some outcomes were similar to those without a heart condition, poor oral health and missed preventive dental care might have additional health implications for children with heart conditions. Among children and adolescents with a heart condition, oral health was notably worse for those with intellectual or developmental disabilities, those living in poverty, and those without insurance. These findings could guide strategies, such as parent and patient education and collaboration between pediatricians, dentists, and cardiologists, to improve oral health and care among children with heart conditions, especially those with fewer resources and intellectual or developmental disabilities.

\section{Acknowledgment}

Ashley Judge.

Corresponding author: Karrie F. Downing, yyx9@cdc.gov, 404-498-0710.

\footnotetext{
${ }^{1}$ Division of Birth Defects and Infant Disorders, National Center on Birth Defects and Developmental Disabilities, CDC; ${ }^{2}$ Division of Oral Health, National Center for Chronic Disease Prevention and Health Promotion, CDC; ${ }^{3}$ Division of Pediatric Cardiology, Department of Pediatrics, Children's Healthcare of Atlanta, Emory University, Atlanta, Georgia.
}

All authors have completed and submitted the International Committee of Medical Journal Editors form for disclosure of potential conflicts of interest. No potential conflicts of interest were disclosed. 


\section{References}

1. Chen MY, Riehle-Colarusso T, Yeung LF, Smith C, Farr SL. Children with heart conditions and their special health care needs-United States, 2016. MMWR Morb Mortal Wkly Rep 2018;67:1045-9. PMID:30260943 https://doi.org/10.15585/mmwr.mm6738a1

2. Wilson W, Taubert KA, Gewitz M, et al.; American Heart Association Rheumatic Fever, Endocarditis, and Kawasaki Disease Committee; American Heart Association Council on Cardiovascular Disease in the Young; American Heart Association Council on Clinical Cardiology; American Heart Association Council on Cardiovascular Surgery and Anesthesia; Quality of Care and Outcomes Research Interdisciplinary Working Group. Prevention of infective endocarditis: guidelines from the American Heart Association: a guideline from the American Heart Association Rheumatic Fever, Endocarditis, and Kawasaki Disease Committee, Council on Cardiovascular Disease in the Young, and the Council on Clinical Cardiology, Council on Cardiovascular Surgery and Anesthesia, and the Quality of Care and Outcomes Research Interdisciplinary Working Group. Circulation 2007;116:1736-54. PMID:17446442 https:// doi.org/10.1161/CIRCULATIONAHA.106.183095

3. Hallett KB, Radford DJ, Seow WK. Oral health of children with congenital cardiac diseases: a controlled study. Pediatr Dent 1992;14:224-30. PMID:1303520

4. Pollard MA, Curzon ME. Dental health and salivary Streptococcus mutans levels in a group of children with heart defects. Int J Paediatr Dent 1992;2:81-5. PMID:1420099 https://doi.org/10.1111/j.1365263X.1992.tb00014.x
5. Franco E, Saunders CP, Roberts GJ, Suwanprasit A. Dental disease, caries related microflora and salivary IgA of children with severe congenital cardiac disease: an epidemiological and oral microbial survey. Pediatr Dent 1996;18:228-35. PMID:8784915

6. Sivertsen TB, Aßmus J, Greve G, Åstrøm AN, Skeie MS. Oral health among children with congenital heart defects in western Norway. Eur Arch Paediatr Dent 2016;17:397-406. PMID:27624134 https://doi. org/10.1007/s40368-016-0243-y

7. Cantekin K, Gumus H, Torun YA, Sahin H. The evaluation of developmental enamel defects and dental treatment conditions in a group of Turkish children with congenital heart disease. Cardiol Young 2015;25:312-6. PMID:24360220 https://doi.org/10.1017/ S1047951113002308

8. Tasioula V, Balmer R, Parsons J. Dental health and treatment in a group of children with congenital heart disease. Pediatr Dent 2008;30:323-8. PMID: 18767512

9. Lebrun-Harris LA, Canto MT, Vodicka P. Preventive oral health care use and oral health status among US children: 2016 National Survey of Children's Health. J Am Dent Assoc 2019;150:246-58. PMID:30922456 https://doi.org/10.1016/j.adaj.2018.11.023

10. Wilson NJ, Lin Z, Villarosa A, George A. Oral health status and reported oral health problems in people with intellectual disability: a literature review. J Intellect Dev Disabil 2019;44:292-304. https://doi.org/10.3 $109 / 13668250.2017 .1409596$ 